\title{
A Fluorogenic Probe for the Copper(I)-Catalyzed Azide-Alkyne Ligation Reaction: Modulation of the Fluorescence Emission via ${ }^{3}\left(n, \pi^{*}\right)-{ }^{1}\left(\pi, \pi^{*}\right)$ Inversion
}

\author{
Zhen Zhou, Christoph J. Fahrni* \\ School of Chemistry and Biochemistry, Georgia Institute of Technology, \\ 770 State Street, Atlanta, GA 30332-0400, U.S.A.
}

\section{Supporting Information}

Materials and Reagents. All chemicals used in the syntheses were purchased from Aldrich. NMR: $\delta$ in ppm vs $\mathrm{SiMe}_{4}\left(0 \mathrm{ppm},{ }^{1} \mathrm{H}, 400 \mathrm{MHz}\right)$. MS: selected peaks; m/z. Melting points are uncorrected. Flash chromatography: Merck silica gel (70-230 mesh). TLC: $0.25 \mathrm{~mm}$, Merck silica gel $60 \mathrm{~F}_{254}$, visualizing at $254 \mathrm{~nm}$ or with $2 \% \mathrm{KMnO}_{4}$ solution.

\section{Synthesis.}

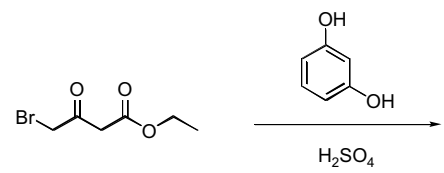

5

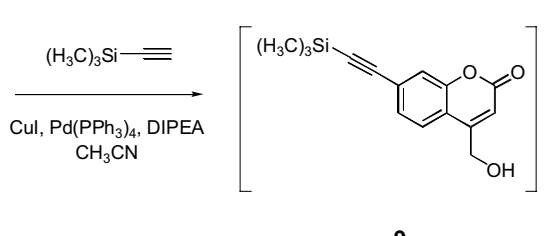

9
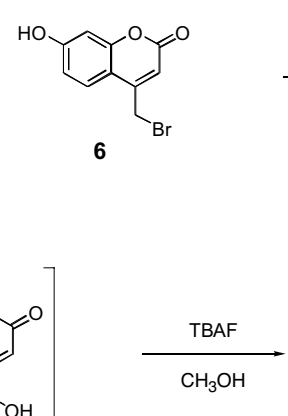

(a) 4-Bromomethyl-7-hydroxy-chromen-2-one (6). A mixture containing 1,3-dihydroxybenzene (220 mg, $2 \mathrm{mmol}$ ), ethyl 4-bromoacetoacetate ${ }^{1} \mathbf{5}$ (420 mg, $2 \mathrm{mmol}$ ), and a catalytic amount of conc. $\mathrm{H}_{2} \mathrm{SO}_{4}$ was stirred at room temperature for $8 \mathrm{~h}$. After addition of water $(50 \mathrm{ml})$ the resulting precipitate was filtered off and dried under vacuum, providing $0.23 \mathrm{~g}$ of bromide 6 as a white solid. (0.90 mmol, 45\% yield). mp 190-192 ${ }^{\circ} \mathrm{C} ;{ }^{1} \mathrm{H}$ NMR (DMSO-d 6 , $\left.400 \mathrm{MHz}\right) \delta 4.80$ (s, 2H), 6.45 (s, 1H), $6.73(\mathrm{~s}, 1 \mathrm{H}), 6.83(\mathrm{~d}, J=8.7,1 \mathrm{H}), 7.69(\mathrm{~d}, J=8.7,1 \mathrm{H}), 10.64(\mathrm{~s}, 1 \mathrm{H}) ; \mathrm{MS}(70 \mathrm{eV}) 254\left(\mathrm{M}^{+}\right.$, 62), 175 (15), 147 (100), 131 (7). EI-HRMS m/e calculated for (M+) $\mathrm{C}_{10} \mathrm{H}_{7} \mathrm{BrO}_{3} 253.9579$ found 253.9583. 
(b) 7-Hydroxy-4-hydroxymethyl-chromen-2-one (7). A suspension of bromide 6 (200 mg, $0.78 \mathrm{mmol}$ ) in $20 \mathrm{ml}$ of water was heated under refluxed for $8 \mathrm{~h}$. After cooling to room temperature the reaction mixture was extracted three times with EtOAc. The combined extracts were dried over anhydrous $\mathrm{MgSO}_{4}$ and concentrated under reduced pressure. The crude product was purified by flash chromatography on silica gel (EtOAc) providing $140 \mathrm{mg}(0.73 \mathrm{mmol}, 94 \%$ yield) of alcohol 7 as white solid. mp $186-188{ }^{\circ} \mathrm{C} ;{ }^{1} \mathrm{H}$ NMR (DMSO-d $\left.\mathrm{d}_{6}, 400 \mathrm{MHz}\right) \delta 4.69(\mathrm{~d}, J=5.6,2 \mathrm{H}), 5.58(\mathrm{t}$, $J=5.6,1 \mathrm{H}), 6.22(\mathrm{~s}, 1 \mathrm{H}), 6.71(\mathrm{~s}, 1 \mathrm{H}), 6.75(\mathrm{~d}, J=8.7,1 \mathrm{H}), 7.50(\mathrm{~d}, J=8.7,1 \mathrm{H}), 10.51(\mathrm{~s}, 1 \mathrm{H}) ; \mathrm{MS}$ (70 eV) $192\left(\mathrm{M}^{+}, 100\right), 164$ (45), 135 (68), 107 (27), 77 (16), 69 (11), 51 (12). EI-HRMS m/e calculated for $(\mathrm{M}+) \mathrm{C}_{10} \mathrm{H}_{8} \mathrm{O}_{4} 192.0423$ found 192.0424 .

(c) 4-Hydroxymethyl-2-oxo-2H-chromen-7-yl trifluoromethanesulfonate (8). A mixture of N-phenyl-bis(trifluoromethanesulfonimide) $(200 \mathrm{mg}, 0.56 \mathrm{mmol})$, alcohol 7 (100 mg, $0.52 \mathrm{mmol}$ ), and $\mathrm{N}, \mathrm{N}$-diisopropylethylamine $(0.12 \mathrm{ml}, 0.69 \mathrm{mmol})$ in $4 \mathrm{ml}$ of $\mathrm{CH}_{3} \mathrm{CN}$ was stirred at room temperature for 2 hours. The solution was diluted with EtOAc and washed three times with water. The combined organic layers were dried over anhydrous $\mathrm{MgSO}_{4}$ and evaporated to dryness. The crude product was purified on silica gel (gradient 10:1 $\rightarrow$ 1:1 hexane:EtOAc ) providing $140 \mathrm{mg}$ of 8 as white solid (0.43 mmol, 83\% yield). mp 112-114 ${ }^{\circ} \mathrm{C} ;{ }^{1} \mathrm{H} \mathrm{NMR}\left(\mathrm{CDCl}_{3}, 400 \mathrm{MHz}\right) \delta 4.91(\mathrm{~s}$, 2H), $6.68(\mathrm{~s}, 1 \mathrm{H}), 7.21(\mathrm{~d}, J=8.8,1 \mathrm{H}), 7.28(\mathrm{~s}, 1 \mathrm{H}), 7.62(\mathrm{~d}, J=8.8,1 \mathrm{H})$; MS (70 eV) $324\left(\mathrm{M}^{+}\right.$, 100), 296 (8), 231 (8), 191 (65), 163 (83), 135 (17), 107 (16), 77 (23), 69 (30). EI-HRMS m/e calculated for $\left(\mathrm{M}^{+}\right) \mathrm{C}_{11} \mathrm{H}_{7} \mathrm{~F}_{3} \mathrm{O}_{6} \mathrm{~S} 323.9915$ found 323.9912

(d) 7-Ethynyl-4-hydroxymethyl-chromen-2-one (10). Trifluoromethanesulfonate 8 (0.25g, $0.77 \mathrm{mmol}$ ) was dissolved in $\mathrm{CH}_{3} \mathrm{CN}(10 \mathrm{ml})$, followed by addition of tetrakis(triphenylphosphine)palladium $(0.09 \mathrm{~g}, 0.078 \mathrm{mmol}), \mathrm{CuI}(0.03 \mathrm{~g}, 0.16 \mathrm{mmol})$, trimethylsilylacetylene $(0.54 \mathrm{ml}, 3.82$ $\mathrm{mmol})$, and N,N-diisopropylethylamine $(0.5 \mathrm{ml}, 2.87 \mathrm{mmol})$. The solution was flushed with argon, degassed by three freeze-thaw cycles, and stirred at room temperature for 36 hours. The mixture was diluted with EtOAc, washed with saturated $\mathrm{NH}_{4} \mathrm{Cl}$ solution, dried over anhydrous $\mathrm{MgSO}_{4}$, and concentrated under reduced pressure. The residue was purified on silica gel (gradient 10:1 $\rightarrow>1: 1$ hexane:EtOAc) providing $177 \mathrm{mg}$ of 7-(trimethylsilylethylethynyl)-4-hydroxylmethylcoumarin 9 
(0.65 mmol, 85\% yield). To remove the silyl protecting group $177 \mathrm{mg}(0.65 \mathrm{mmol})$ of crude 9 was dissolved in methanol $(25 \mathrm{ml})$, and tetrabutylammomium fluoride $(2 \mathrm{ml} 1.0 \mathrm{M}$ solution in THF, 2.0

mmol) was added. The reaction mixture was stirred at $60^{\circ} \mathrm{C}$ for $15 \mathrm{~min}$. After cooling to room temperature, water was added until precipitation occurred. The product was filtered off and purified on silica gel (50:50 hexane/EtOAc) providing $94 \mathrm{mg}$ of $\mathbf{1 0}$ as white solid (0.47 mmol, 72\% yield). $\mathrm{mp}$ 179-181 ${ }^{\circ} \mathrm{C} ;{ }^{1} \mathrm{H}$ NMR (DMSO-d $\left.6,400 \mathrm{MHz}\right) \delta 4.52(\mathrm{~s}, 1 \mathrm{H}), 4.75(\mathrm{~d}, J=5.1,2 \mathrm{H}), 5.71(\mathrm{t}, J=5.1$, 1H), $6.47(\mathrm{~s}, 1 \mathrm{H}), 7.41(\mathrm{~d}, J=8.1,1 \mathrm{H}), 7.53(\mathrm{~s}, 1 \mathrm{H}), 7.68(\mathrm{~d}, J=8.1,1 \mathrm{H}) ; \mathrm{MS}(70 \mathrm{eV}) 200\left(\mathrm{M}^{+}\right.$, 100), 172 (35), 155 (20), 143 (22), 115 (40), 63 (12). EI-HRMS m/e calculated for $\left(\mathrm{M}^{+}\right) \mathrm{C}_{12} \mathrm{H}_{8} \mathrm{O}_{3}$ 200.0473 found 200.0479 .

(e) Succinic acid mono-(7-ethynyl-2-oxo-2H-chromen-4-ylmethyl) ester (1). A solution of 9 (20mg, $0.1 \mathrm{mmol})$, succinic anhydride (12 mg, $0.12 \mathrm{mmol})$, KOAc (20 mg, $0.2 \mathrm{mmol})$ in $\mathrm{CH}_{3} \mathrm{CN}$ was stirred at $40^{\circ} \mathrm{C}$ for $2 \mathrm{~h}$. The resulting reaction mixture was diluted with EtOAc $(50 \mathrm{~mL})$, washed with $1 \mathrm{M} \mathrm{HCl}(5 \mathrm{ml})$, dried over anhydrous $\mathrm{MgSO}_{4}$, and concentrated under reduced pressure. The residue $(30 \mathrm{mg})$ was washed with $\mathrm{MeOH}(2 \mathrm{~mL})$ and dried under vacuum providing $20 \mathrm{mg}$ (0.067 mmol, 67\% yield) of fluorophore 1 as pale yellow solid. For spectroscopic characterization an analytical sample was further purified by flash chromatography (1:5 methanol:EtOAc). $\mathrm{mp}>190^{\circ} \mathrm{C}$ (dec.); ${ }^{1} \mathrm{H}$ NMR (DMSO-d $\left.{ }_{6}, 400 \mathrm{MHz}\right) \delta 2.54$ (t, J=6.9, 2H), 2.69 (t, $J=6.9,2 \mathrm{H}), 4.53(\mathrm{~s}, 1 \mathrm{H}), 5.39(\mathrm{~s}, 2 \mathrm{H}), 6.50(\mathrm{~s}, 1 \mathrm{H}), 7.43(\mathrm{~d}, J=8.2,1 \mathrm{H}), 7.54(\mathrm{~s}, 1 \mathrm{H}), 7.72(\mathrm{~d}$, $J=8.2,1 \mathrm{H}) ; \mathrm{MS}(70 \mathrm{eV}) 301\left([\mathrm{M}+1]^{+}, 100\right)$. ESI-TOF-HRMS $m / e$ calculated for $[\mathrm{M}+1]^{+}$ $\mathrm{C}_{16} \mathrm{H}_{13} \mathrm{O}_{6} 301.0712$ found 301.0723 .

\section{(f) Succinic acid mono-\{7-[1-(4-carboxy-benzyl)-1H-[1,2,3]triazol-4-yl]-2-oxo-2H-} chromen-4-ylmethyl\}ester (3). (Preparative Huisgen coupling) A solution containing fluorophore 1 (20mg, $0.067 \mathrm{mmol})$, azide 2 (11.8mg, $0.067 \mathrm{mmol}), \mathrm{CuSO}_{4}(1.1 \mathrm{mg}, 0.0068 \mathrm{mmol})$ and ascorbic acid $(11.8 \mathrm{mg}, 0.067 \mathrm{mmol})$ in $2 \mathrm{ml} 50: 50 t-\mathrm{BuOH} / \mathrm{H}_{2} \mathrm{O}$ mixture was stirred at room temperature for 2 days. After addition of water $(10 \mathrm{~mL})$ the precipitated product was collected by filtration, washed with little $\mathrm{MeOH}$, and dried under vacuum providing $24 \mathrm{mg}$ of coupling product 3 as white solid (0.051 mmol, 76\% yield). mp $>245{ }^{\circ} \mathrm{C}$ (dec.); ${ }^{1} \mathrm{H}$ NMR (DMSO-d $\left.6,400 \mathrm{MHz}\right) 2.56(\mathrm{t}, J=6.7,2 \mathrm{H})$, 
$2.71(\mathrm{t}, J=6.7,2 \mathrm{H}), 5.43(\mathrm{~s}, 2 \mathrm{H}), 5.79(\mathrm{~s}, 2 \mathrm{H}), 6.48(\mathrm{~s}, 1 \mathrm{H}), 7.46(\mathrm{~d}, J=7.0,2 \mathrm{H}), 7.80-8.00(\mathrm{~m}, 5 \mathrm{H})$, 8.89 (s, 1H); MS (70 eV) $478\left([\mathrm{M}+1]^{+}, 100\right), 301$ (5), 279 (12). ESI-HRMS m/e calculated for $(\mathrm{M}+\mathrm{H}) \mathrm{C}_{24} \mathrm{H}_{20} \mathrm{~N}_{3} \mathrm{O}_{8} 478.1285$ found 478.1250.

(g) 4-Azidomethyl benzoic aicd 2. A solution of 4-chloromethyl benzoic acid (300 mg, $1.76 \mathrm{mmol})$ and $\mathrm{NaN}_{3}(300 \mathrm{mg}, 4.6 \mathrm{mmol})$ in $10 \mathrm{ml}$ of abs. EtOH was refluxed overnight. After cooling to room temperature $10 \mathrm{ml}$ of water and three drops of aq. $\mathrm{HCl}(37 \%)$ were added. The resulting precipitate was filtered off, washed with water, and dried under reduced pressure providing $220 \mathrm{mg}$ (1.24 mmol, 70\% yield) of azide 2 as white solid. mp $129-131{ }^{\circ} \mathrm{C} ;{ }^{1} \mathrm{H}$ NMR (DMSO-d 6 , $400 \mathrm{MHz}) 4.54(\mathrm{~s}, 2 \mathrm{H}), 7.46(\mathrm{~d}, J=8.1,2 \mathrm{H}), 7.93(\mathrm{~d}, J=8.1,2 \mathrm{H}) ; \mathrm{MS}(70 \mathrm{eV}) 177\left(\mathrm{M}^{+}, 49\right), 149$ (91), 135 (100), 107 (31), 104 (25), 77 (36), 51 (23). EI-HRMS m/e calculated for $\left(\mathrm{M}^{+}\right) \mathrm{C}_{8} \mathrm{H}_{7} \mathrm{~N}_{3} \mathrm{O}_{2}$ 177.0538 found 177.0552 .

(h) Ligand 4. A solution of tripropargylamine (100 mg, $0.76 \mathrm{mmol})$, ethyl azidoacetate (294 $\mathrm{mg}, 2.28 \mathrm{mmol}),\left[\mathrm{Cu}\left(\mathrm{CH}_{3} \mathrm{CN}\right)_{4}\right] \mathrm{PF}_{6}(10 \mathrm{mg}, 0.027 \mathrm{mmol})$, and $\mathrm{N}$, N-diisopropylethylamine $(0.4 \mathrm{ml}$, $2.29 \mathrm{mmol}$ ) in $3 \mathrm{ml}$ of $\mathrm{CH}_{3} \mathrm{CN}$ was stirred at room temperature for 2 days. The reaction mixture was concentrated under reduced pressure, and the residue was diluted with $\mathrm{CH}_{2} \mathrm{Cl}_{2}$ and washed with water. The organic phase was dried with anhydrous $\mathrm{MgSO}_{4}$, and concentrated. The crude product was purified on silica gel $\left(\mathrm{CH}_{2} \mathrm{Cl}_{2}-\mathrm{MeOH} 10: 1\right)$ providing $392 \mathrm{mg}(0.76 \mathrm{mmol}$, quantitative yield)of ligand 4 as light yellow oil. ${ }^{1} \mathrm{H} \mathrm{NMR}\left(\mathrm{CDCl}_{3}, 400 \mathrm{MHz}\right) \delta 1.28(\mathrm{t}, J=7.1,9 \mathrm{H}), 3.79(\mathrm{~s}, 6 \mathrm{H}), 4.30$ $(\mathrm{q}, J=7.1,6 \mathrm{H}), 5.17(\mathrm{~s}, 6 \mathrm{H}), 7.84(\mathrm{~s}, 3 \mathrm{H}) ; \mathrm{MS}(70 \mathrm{eV}) 519\left([\mathrm{M}+1]^{+}, 100\right), 390(8), 279$ (8). ESITOF-HRMS m/e calculated for $[\mathrm{M}+1]^{+} \mathrm{C}_{21} \mathrm{H}_{31} \mathrm{~N}_{10} \mathrm{O}_{6} 519.2403$ found 519.2422.

Steady-state Absorption and Fluorescence Spectroscopy: All sample stock solutions and buffer solutions were filtered through $0.2 \mu \mathrm{m}$ Nylon membrane filters to remove interfering dust particles or fibers. UV-vis absorption spectra were recorded at $25^{\circ} \mathrm{C}$ using a Varian Cary Bio50 UV-vis spectrometer with constant-temperature accessory. Fluorescence emission and excitation spectra were recorded with a PTI fluorimeter and FELIX software. The path length was $1 \mathrm{~cm}$ with a cell volume of $3.0 \mathrm{~mL}$. All fluorescence spectra have been corrected for the spectral response of the detection system (emission correction file provided by instrument manufacturer) and for the 
spectral irradiance of the excitation channel (via calibrated photodiode). Quantum yields were determined using quinine sulfate dihydrate in $1.0 \mathrm{~N} \mathrm{H}_{2} \mathrm{SO}_{4}\left(\Phi_{\mathrm{f}}=0.543 \pm 0.03\right)$ as fluorescence standard. ${ }^{2}$

Fluorescence Lifetime Measurements: Fluorescence lifetime data (Figure S1 and S2) were acquired with a Photon Technology International (PTI) fluorescence lifetime instrument (resolution $50 \mathrm{ps}$ ). The system uses a nitrogen laser (model GL-3300) as excitation source combined with a stroboscopic detector. All samples were excited at $337 \mathrm{~nm}$ with a repetition rate of $10 \mathrm{~Hz}$ (pulse width $2 \mathrm{~ns}$ ) and the fluorescence signal was analyzed after passing through a monochromator set at $400 \mathrm{~nm}$. The data were analyzed with the TimeMaster Pro software package provided with the spectrometer.
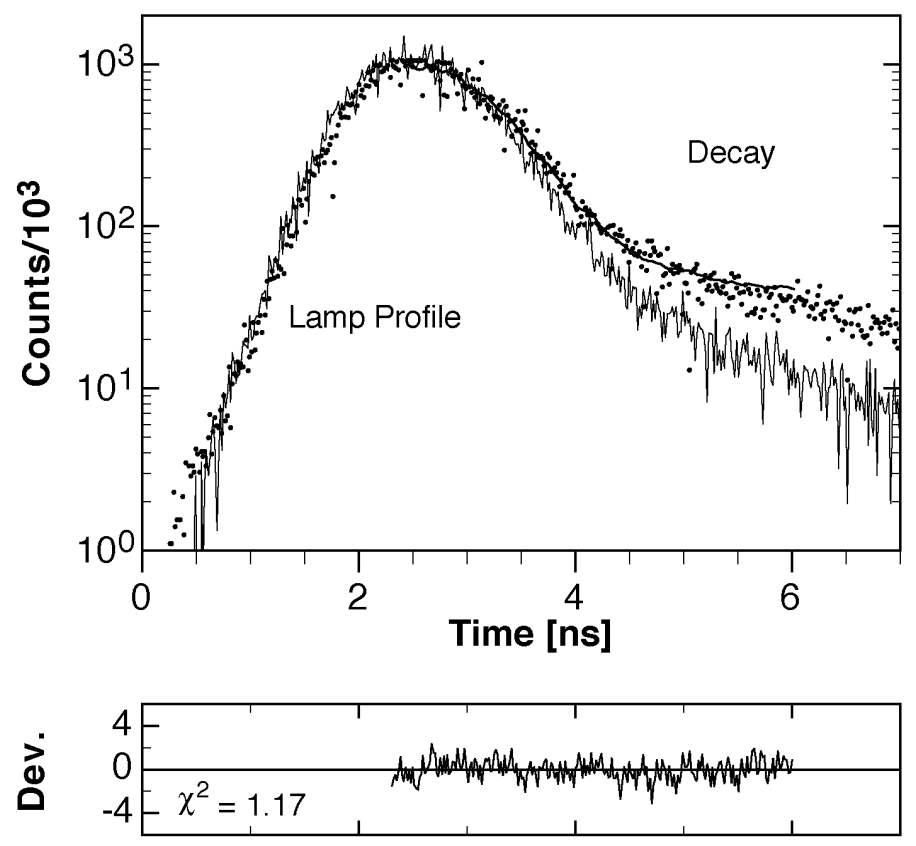

Figure $\mathbf{S} 1$ Fluorescence decay data for coumarin 1 in aqueous solution $(\mathrm{pH}$ 7.20, $10 \mathrm{mM}$ PIPES, 0.1M KCl) 

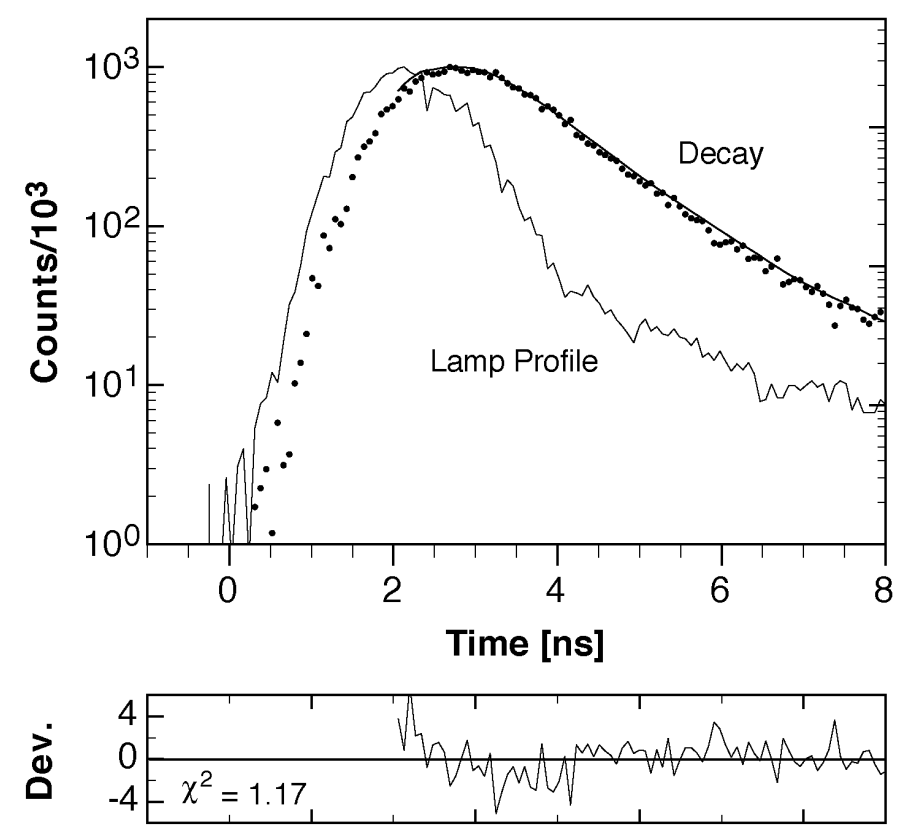

Figure S2: Fluorescence decay data for coumarin 3 in aqueous solution ( $\mathrm{pH} 7.20,10 \mathrm{mM}$ PIPES, $0.1 \mathrm{M} \mathrm{KCl})$

Kinetic Measurements by Fluorescence Spectroscopy: Conditions (1): A solution containing fluorophore $1(60 \mu \mathrm{M})$, azide $2(20 \mu \mathrm{M}), \mathrm{CuSO}_{4}(60 \mu \mathrm{M})$, and tris-triazole ligand $4(60$ $\mu \mathrm{M})$ in $2.5 \mathrm{~mL}$ of buffer solution (10 mM PIPES, $\mathrm{pH} 7.2)$ was stirred at $37^{\circ} \mathrm{C}$ for $5 \mathrm{~min}$. After addition of ascorbic acid $(300 \mu \mathrm{M})$ the fluorescence emission intensity at $420 \mathrm{~nm}\left(\lambda_{\mathrm{ex}} 350 \mathrm{~nm}\right)$ was monitored in $5 \mathrm{~min}$ intervals. For each data point the fluorescence intensity was measured over a period of $5 \mathrm{sec}$ and averaged over a total of 5 points. In a control experiment the same conditions were used except that azide $\mathbf{2}$ was not added to the solution. Conditions (2): Procedure identical with conditions (1), but using the following concentrations: fluorophore $1(5 \mu \mathrm{M})$, azide 2 (100 $\mu \mathrm{M}), \mathrm{CuSO}_{4}(60 \mu \mathrm{M})$, and tris-triazole ligand $4(60 \mu \mathrm{M})$, ascorbic acid $(300 \mu \mathrm{M})$. The fluorescence emission was acquired in 10 seconds intervals.

Computational Methods: The geometry of the ground state structures were optimized by the density functional method using the Q-Chem ${ }^{3}$ electronic structure calculation suite of programs. Becke's gradient corrected three-parameter exchange functional with the correlation functional of Lee, Yang, and Parr (B3LYP) ${ }^{4}$ and the split-valence polarized 6-31G* $(6-31 \mathrm{G}(\mathrm{d}))$ basis set were 
used. The gas-phase and solution-phase vertical excitation energies were calculated using Zerner's INDO S/CI model ${ }^{5}$ using the ArgusLab software package, ${ }^{6}$ which also includes the implementation of a SCRF solvent model. ${ }^{7}$ Ground- and excited-state dipole moments were also calculated on the basis of the INDO S/CI model. Molecular orbitals were visualized with the ArgusLab software package. The coordinates of the geometry-optimized structures are provided with Tables S1 and S2. 
Table S1: Cartesian atomic coordinates for the geometry optimized structure of coumarin 1 (B3LYP/6-31G*).

\begin{tabular}{crrr}
\hline & \multicolumn{1}{c}{$\mathrm{x} / \AA$} & \multicolumn{1}{c}{$\mathrm{y} / \AA$} & \multicolumn{1}{c}{$\mathrm{I} / \mathrm{A}$} \\
\hline $\mathrm{C}$ & 1.973437 & -1.353509 & -0.000136 \\
$\mathrm{C}$ & 2.332568 & 0.013403 & 0.000030 \\
$\mathrm{C}$ & 1.326195 & 0.988121 & 0.000231 \\
$\mathrm{C}$ & -0.011946 & 0.600005 & 0.000231 \\
$\mathrm{C}$ & -0.387965 & -0.758797 & 0.000061 \\
$\mathrm{C}$ & 0.639360 & -1.722696 & -0.000141 \\
$\mathrm{O}$ & -0.936381 & 1.602268 & 0.000447 \\
$\mathrm{C}$ & -2.309452 & 1.349136 & 0.000191 \\
$\mathrm{C}$ & -2.699386 & -0.053853 & 0.000142 \\
$\mathrm{C}$ & -1.807470 & -1.076353 & 0.000077 \\
$\mathrm{C}$ & -2.262687 & -2.511216 & -0.000023 \\
$\mathrm{C}$ & 3.708037 & 0.396895 & 0.000026 \\
$\mathrm{O}$ & -3.058099 & 2.296865 & -0.000703 \\
$\mathrm{C}$ & 4.874346 & 0.718030 & -0.000216 \\
$\mathrm{H}$ & 2.753657 & -2.107094 & -0.000290 \\
$\mathrm{H}$ & 1.570780 & 2.044198 & 0.000375 \\
$\mathrm{H}$ & 0.379101 & -2.776212 & -0.000312 \\
$\mathrm{H}$ & -3.769529 & -0.229385 & 0.000050 \\
$\mathrm{H}$ & -1.888352 & -3.044462 & -0.882482 \\
$\mathrm{H}$ & -1.888072 & -3.044708 & 0.882166 \\
$\mathrm{H}$ & -3.353653 & -2.575045 & 0.000129 \\
$\mathrm{H}$ & 5.901687 & 1.004644 & -0.000419 \\
& & & \\
\hline
\end{tabular}


Table S2: Cartesian atomic coordinates for the geometry optimized structure of coumarin $\mathbf{3}$ (B3LYP/6-31G*).

\begin{tabular}{crrr}
\hline & \multicolumn{1}{c}{$\mathrm{x} / \AA$} & \multicolumn{1}{c}{$\mathrm{y} / \AA$} \\
\hline $\mathrm{Ctom}$ & -0.462485 & -1.577270 & 0.000092 \\
$\mathrm{C}$ & -0.932068 & -0.247576 & 0.000154 \\
$\mathrm{C}$ & -0.004633 & 0.798521 & 0.000179 \\
$\mathrm{C}$ & 1.359629 & 0.516906 & 0.000126 \\
$\mathrm{C}$ & 1.846007 & -0.806342 & 0.000093 \\
$\mathrm{C}$ & 0.895740 & -1.846052 & 0.000058 \\
$\mathrm{C}$ & 2.202911 & 1.589787 & 0.000092 \\
$\mathrm{O}$ & 3.589881 & 1.448395 & -0.000157 \\
$\mathrm{C}$ & 4.093097 & 0.081728 & -0.000084 \\
$\mathrm{C}$ & 3.285440 & -1.008887 & 0.000013 \\
$\mathrm{C}$ & 3.854078 & -2.402586 & -0.000196 \\
$\mathrm{C}$ & -2.366269 & 0.052761 & 0.000183 \\
$\mathrm{C}$ & 4.261152 & 2.453302 & -0.000162 \\
$\mathrm{O}$ & -3.461586 & -0.795289 & 0.000447 \\
$\mathrm{C}$ & -1.167685 & -2.403127 & 0.000055 \\
$\mathrm{H}$ & -0.338377 & 1.829660 & 0.000213 \\
$\mathrm{H}$ & 1.233863 & -2.877536 & -0.000034 \\
$\mathrm{H}$ & 5.174046 & -0.007789 & -0.000094 \\
$\mathrm{H}$ & 3.521692 & -2.964788 & 0.881037 \\
$\mathrm{H}$ & 3.524316 & -2.963269 & -0.883410 \\
$\mathrm{H}$ & 4.946783 & -2.380027 & 0.001427 \\
$\mathrm{H}$ & -3.561818 & -1.869706 & 0.000671 \\
$\mathrm{H}$ & -2.840235 & 1.339035 & 0.000182 \\
$\mathrm{~N}$ & -4.139688 & 1.326450 & -0.000104 \\
$\mathrm{~N}$ & -4.534771 & 0.030001 & -0.000022 \\
$\mathrm{~N}$ & -5.950514 & -0.295571 & -0.000534 \\
$\mathrm{C}$ & -6.212890 & -0.868570 & -0.894815 \\
$\mathrm{H}$ & -6.213147 & -0.869982 & 0.892756 \\
$\mathrm{H}$ & -6.494335 & 0.649583 & 0.000122 \\
$\mathrm{H}$ & & & \\
\hline & & &
\end{tabular}

\section{References:}

(1) Choi, H. Y.; Chi, D. Y. Org. Lett. 2003, 5, 411.

(2) Demas, J. N.; Crosby, G. A. J. Phys. Chem. 1971, 75, 991.

(3) Kong, J.; White, C. A.; Krylov, A. I.; Sherrill, C. D.; Adamson, R. D.; Furlani, T. R.; Lee, M. S.; Lee, A. M.; Gwaltney, S. R.; Adams, T. R.; Ochsenfeld, C.; Gilbert, A. T. B.; Kedziora, G. S.; Rassolov, V. A.; Maurice, D. R.; Nair, N.; Shao, Y.; Besley, N. A.; Maslen, P. E.; J. P. Dombroski; Dachsel, H.; Zhang, W. M.; Korambath, P. P.; Baker, J.; Byrd, E. F. C.; Voorhis, T. V.; Oumi, M.; Hirata, S.; Hsu, C. P.; Ishikawa, N.; Florian, J.; Warshel, A.; Johnson, B. G.; Gill, P. M. W.; Head-Gordon, M.; Pople, J. A. Q-Chem; 2.0 ed.; Q-Chem Inc.: PA, 2000.

(4) Becke, A. D. J. Chem. Phys. 1993, 98, 5648; Lee, C. T.; Yang, W. T.; Parr, R. G. Phys. Rev. B 1988, 37, 785.

(5) Bacon, A. D.; Zerner, M. C. Theor. Chim. Acta 1979, 53, 21.

(6) Thompson, M. A. ArgusLab 3.1; Planaria Software LLC, Seattle, WA, http://www.arguslab.com, 2003.

(7) Karelson, M. M.; Zerner, M. C. J. Phys. Chem. 1992, 96, 6949. 